\title{
A catalogue of the observations of the mutual phenomena of the Galilean satellites made in 1991 during the PHEMU91 campaign
}

J.E. Arlot ${ }^{1}$, C. Ruatti ${ }^{1}$, W. Thuillot ${ }^{1}$, J. Arsenijevic ${ }^{26}$, R. Baptista ${ }^{2}$, J. Barroso Jr. ${ }^{2}$, C. Bauer ${ }^{33}$, J. Berthier ${ }^{1}$, C. Blanco ${ }^{3}$, P. Bouchet ${ }^{4}$, J. Bourgeois ${ }^{5}$, H.J.J. Bulder ${ }^{6}$, R. Burchi ${ }^{7}$, J.A. Cano ${ }^{16}$, R. Casas ${ }^{8}$, F. Chauvet $^{10}$, D. Chis $^{9}$, F. Colas ${ }^{1}$, J. Colin ${ }^{10}$, V. D'Ambrosio ${ }^{7,11}$, G. De Angelis ${ }^{1,7}$, G. De Benedetto ${ }^{11}$, H. Denzau ${ }^{13}$, J.M. Desbats ${ }^{10}$, P. Descamps ${ }^{1}$, A. Dipaolantonio ${ }^{7}$ A. Dumitrescu ${ }^{25}$ L. Farcas ${ }^{35}$, M. Federspiel ${ }^{14}$, T. Flatrès ${ }^{27}$, M. Froeschlé ${ }^{15}$, O. Gherega ${ }^{35}$, J.M. Gomez-Forrellad ${ }^{16}$, J. Guarro ${ }^{16}$, O. Hainaut ${ }^{4}$, A. Horvat ${ }^{35}$, G. Helmer ${ }^{15}$, D. Hube ${ }^{17}$, Y. Ito ${ }^{18}$, M. Kidger ${ }^{8}$, J. Lecacheux ${ }^{19}$, J.F. Le Campion ${ }^{10}$, J.C. Le Floch ${ }^{20}$, A. Mallama ${ }^{21}$, B.E. Martin ${ }^{17}$, J.F. Mellilo ${ }^{22}$, C. Meyer ${ }^{15}$, S. Molau $^{23}$, G. Montignac ${ }^{10}$, B. Morando ${ }^{1}$, B. Nicolet $^{24}$, B. Nitschelm ${ }^{24}$, G. Oprescu ${ }^{25}$, A. Piersimoni ${ }^{7}$, D. Przewozny ${ }^{23}$, V. Protitch-Benishek ${ }^{26}$, M. Rapaport ${ }^{10}$, A. Riou ${ }^{27}$, J.J. Sacré ${ }^{27}$, F. Sèvre ${ }^{28}$, V. Shkodrov ${ }^{29}$, J. Souchay ${ }^{18}$, H. Takami ${ }^{30}$, R. Taylor ${ }^{33}$, D.J. Tholen ${ }^{31}$, V. Turcu ${ }^{9}$, R. Vasundhara ${ }^{32}$, J. Vidal ${ }^{16}$, D.T. Vu ${ }^{1}$, G. White ${ }^{33}$, and R.P. Wilds ${ }^{34}$

${ }^{1}$ Bureau des longitudes, URA 707 du CNRS, 77 avenue Denfert-Rochereau, 75014 Paris, France; ${ }^{2}$ Observatorio Nacional, Rio de Janeiro, Brasil; ${ }^{3}$ Istituto di Astronomia, Catania, Italy; ${ }^{4}$ European Southern Observatory, La Silla, Chile; ${ }^{5}$ Reux, Belgique; ${ }^{6}$ Zoetermeer, The Netherlands; ${ }^{7}$ Osservatorio di Collurania, Teramo, Italy; ${ }^{8}$ Instituto Astrofisico di Canarias, Teneriffe, Spain; ${ }^{9}$ Observatorul Astronomic Cluj, Cluj Napoca, Romania; ${ }^{10}$ Observatoire de Bordeaux, France; ${ }^{11}$ Roseto degli Abruzzi, Teramo, Italy; ${ }^{12}$ GAR, Reggio Calabria, Italy; ${ }^{13}$ Essen, Germany; ${ }^{14}$ Heuweiler, Germany; ${ }^{15}$ Observatoire de la Côte d'Azur, France; ${ }^{16}$ GEA, Barcelona, Spain; ${ }^{17}$ Department of Physics, University of Alberta, Canada; ${ }^{18}$ SendaiCity, Myagi, Japan; ${ }^{19}$ Observatoire de Paris, France; ${ }^{20}$ Clichy, France; ${ }^{21}$ Hughes STX Corporation, Lanham Md, U.S.A.; ${ }^{22}$ ASL, Holtsville NY, U.S.A.; ${ }^{23}$ Astronomical Observatory, Berlin, Germany; ${ }^{24}$ Observatoire de Genève, Suisse; ${ }^{25}$ Institutul Astronomic, Bucuresti, Romania; ${ }^{26}$ Observatoire de Belgrade, Yougoslavie; ${ }^{27}$ La Primaudière, France; ${ }^{28}$ IAP, Paris, France; ${ }^{29}$ Academy of Sciences, Sofia, Bulgaria; ${ }^{30}$ CRL, Tokyo, Japan; ${ }^{31}$ Institute of Astronomy, Hawaii University, U.S.A.; ${ }^{32}$ Institute of Astrophysics, Bangalore, India; ${ }^{33}$ University of Western Sydney, Nepean, Sydney, Australia; ${ }^{34}$ NEKAAL, Topeka, Kansas, U.S.A.; ${ }^{35}$ Astronomical Institute, Timisoara, Romania

Received April 10, 1996; accepted January 14, 1997

\begin{abstract}
In this paper, all the light-curves obtained during the PHEMU91 campaign of observations of the mutual phenomena of the Galilean satellites are presented. These observations give accurate astrometric positions of major interest for dynamical studies of the motion of the Galilean satellites. The aim of this work is to give observational data directly usable for theoretical studies. We made 374 observations of 111 mutual events from 56 sites. The corresponding data are given in this paper ${ }^{1}$. The accuracy of each observation has been deduced from a comparison with the theoretical predictions. For each observation, information is given about the telescope, the receptor, the site and the observational conditions.
\end{abstract}

Send offprint requests to: J.-E. Arlot

1 Tables 3 and figure 3 are available in electronic form at the CDS via anonymous ftp to cdsarc.u-strasbg.fr (130.79.128.5) or via http://cdsweb.u-strasbg.fr/Abstract.html
Key words: Jupiter - Galilean satellites occultations - astrometry

\section{Introduction}

For the favorable occurrence of mutual phenomena of the Galilean satellites in 1991, a special effort has been made in order to observe as many as possible events. A campaign of observation has been coordinated among 56 sites worldwide. Several techniques were used for these observations. A few observations were also made visually by amateur astronomers. They are presented in this paper only for the qualitative information that they provide for comparison with the other lightcurves. The whole set of observations is composed of 374 lightcurves of 111 events. 


\section{The mutual events}

Once every six years, the Earth and the Sun cross the equatorial plane of Jupiter (the jovian declinations of the Earth and the Sun become zero). Since the Galilean satellites have their orbital planes very close to the jovian equator, mutual phenomena occur: the satellites occult and eclipse each other during a period of about six months. The 1991 period (Arlot 1990) was very favorable since it happened during the opposition of Jupiter and the Sun (Fig. 1). Northern hemisphere observatories were favored since the declination of Jupiter was positive.

Since there is no atmosphere around the Galilean satellites, the photometric observations of such phenomena are very accurate for astrometric purposes. The relative positions of the satellites involved may be obtained with an accuracy better than the one obtained by the other kinds of observation: $200 \mathrm{~km}$ for the best photographic observations and $700 \mathrm{~km}$ for the eclipses by Jupiter observed visually. Note that CCD photometry of eclipses by Jupiter should reach an accuracy of $40 \mathrm{~km}$ according to Mallama et al. (1994).

Such an accuracy is necessary for several reasons. First, the orbital motions of the Galilean satellites have to be very well known in order to prepare the missions of the space probes exploring the jovian system. Second, all the theoretical problems related to the motion of the Galilean satellites are not solved. This motion, which is affected by numerous perturbations, is one of the most complex in celestial mechanics. The theoretical data presented in this paper are calculated using ephemerides G-5 (Arlot 1982) based on the work of Lieske (1977). These ephemerides need improvements. For example, Io is suspected of having a secular acceleration due to energy dissipation (Goldstein \& Jacobs 1986; Lieske 1987). Such an effect is very small and observations during several series of mutual events will be needed in order to determine the acceleration.

Coordinated campaigns of observations are very useful for getting a large amount of data. All the events occur in a small interval of time; so, numerous observers observing from several sites are necessary. To get as many events as possible, it is necessary to observe from different longitudes (to get different events) and from several sites (to avoid meteorological problems). In 1979, the conditions were not favorable because of the conjunction of Jupiter with the Sun, so, very few observations were obtained (Arlot et al. 1982). Contrarily, in 1985 the conditions were very favorable and we got 154 lightcurves from 63 sites in Europe and South America. Observations were easier in the Southern hemisphere because of the negative declination of Jupiter (Arlot et al. 1992).

\section{The PHEMU91 campaign}

Table 1 gives the list of the names, longitudes and latitudes of the sites from which the mutual events were ob-

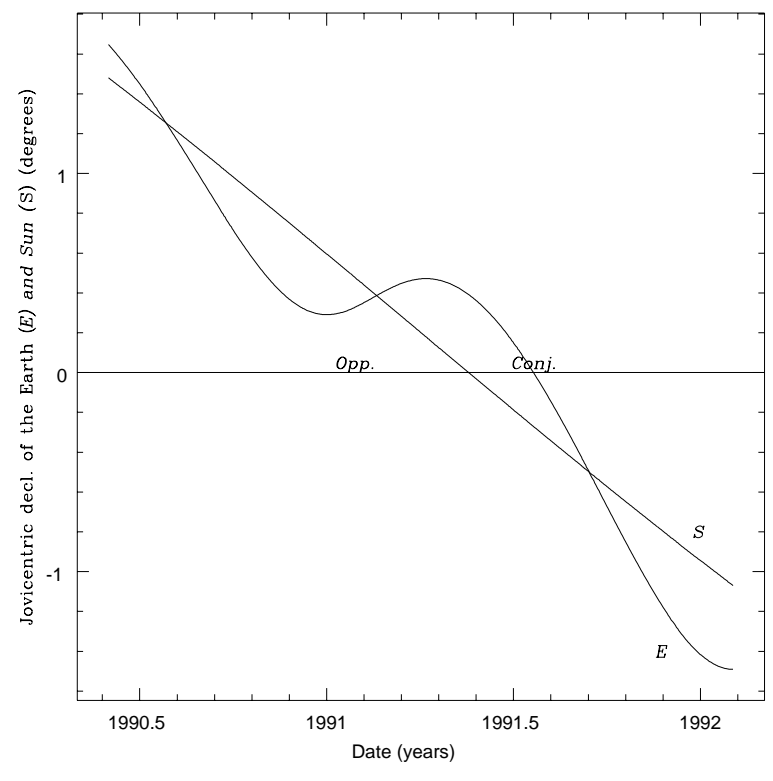

Fig. 1. Jovicentric declinations of the Earth and the Sun during the 1991 occurrence

served and Table 2 gives the list of the receptors used for the recording of the observations. Several kinds of receptors were used for the observations:

1. photometric receptors,

2. two-dimensional receptors,

3. visual observations.

They were used with reflector and refractor telescopes, many of which had small apertures since the Galilean satellites are about magnitude 5. A filter (specified in the data) was used in most cases.

\subsection{Photometric receptors}

These receptors are currently used for absolute photometry. In the case of the mutual events, only relative photometry is necessary. Anyway, since the elevation of Jupiter above the horizon may be very small, absolute photometry is not possible: the air mass is often too large. The description of some photometers that we used is as follows:

1. At Paris Observatory (France), behind a $38 \mathrm{~cm}-$ refractor, an uncooled RCA 4840 photomultiplier (multialcali photocathode) the sensitivity of which is from 350 to 750 micrometers. This equipment is described by Briot (1987) and will be denoted "PM9" in the data tables.

2. At CERGA (Observatory at Calern near Grasse, France) behind a $150 \mathrm{~cm}$ reflector, the photometer TELOC at 450 micrometers, described by Froeschlé et al. (1988) and denoted "POB" for channel $\mathrm{B}$, "POV" for channel V, "POR" for channel $\mathrm{R}$ in the data tables.

3. At Teramo Observatory (Italy), behind either a $40 \mathrm{~cm}$ refractor or a $50 \mathrm{~cm}$ reflector, an EMI 6256A or a 
6256SA described by Burchi \& Di Paolantonio (1987) and denoted "PM4" in the data tables.

4. At Catania Observatory (Italy), behind a $91 \mathrm{~cm}-$ reflector, a cooled photon counting single-head photometer equipped with an EMI 9789QA photomultiplier described by Blanco et al. (1991) and denoted "PM2" in the data tables.

5. At GEA (Grup d'Estuds Astronomics) several telescopes were used the apertures of which varied from 20 to $41 \mathrm{~cm}$. Two types of photometers were used: either a photodiode photometer OPTEC-SSP3 denoted "PM5" in the data tables, either a photomultiplier HPO (1P21) or an OPTEC SSP-5 (Hamamatsu R1414) denoted "PM17" in the data tables. The telescopes were used either in the Pyrenean mountains or in the vicinity of Barcelona (Spain). The description of the equipment is given by Gomez-Forrellad (1987).

6. At the European Southern Observatory (La Silla, Chile), behind either a $50 \mathrm{~cm}$ or a $1 \mathrm{~m}$ reflector, two photometers were used: either a EMI 6256(S-13) or a Quantacon RCA-31036 (Ga-As) denoted "PM4" and "PM3" in the data tables. The description is given by Gouiffes et al. (1987).

7. At Brasopolis Observatory (Brazil), behind a $60 \mathrm{~cm}$ reflector, a cooled Hamamatsu 943-02 denoted "PM15" in the data tables.

8. At Mauna Kea (Hawaii, U.S.A.), a dry ice cooled RCA C31034A photomultiplier behind a $61 \mathrm{~cm}$-reflector denoted PM16. The filter was a $\mathrm{H} 2 \mathrm{O}+$ from the IHW filter set.

9. At Jungfrau Observatory (Switzerland), behind a $76 \mathrm{~cm}$ telescope, a cooled photomultiplier photometer (with a S11 cathode) denoted "S11" in the data tables.

10. Some amateur astronomers used also photometers. At Zoetermeer (Netherlands), an OPTEC photodiode photometer was used (denoted "PM14" in the data tables), as well as at Essen (Germany) and Holtsville (U.S.A.) where photodiode photometers denoted "PM5" were used.

The data tables, give also the nature of the filter used as well as the observational conditions for each event.

\subsection{Two-dimensional receptors}

Another interesting type of receptors is the twodimensional ones. In fact, these receptors record images in place of light-flux. Depending on the receptor, it is possible to calculate the light-flux of the satellites during the event. However, several problems have to be solved. The most important is the speed of acquisition of the images. The time constant depends on the duration of the event but should be, most of time, less than one second of time. Some receptors are not able to acquire images so fast. The receptors that we used are as follows:

1. cooled CCD (c.) driven by a computer; images are directly recorded as numerical arrays of pixels and the calculation of the light-flux, the reduction using reference objects and the sky background is easy. However, the time constant is often large and we will get a small number of points for the light-curves. For long events, it will be very favorable since the noise will decrease. We note that faster computers and better softwares will permit much higher frequency acquisition for the next campaigns of observations. The receptors used are denoted "CCD1" for the one built by Bordeaux Observatory and described by Le Campion et al. (1992), "CCD2" for the one built by Pic-du-Midi Observatory and described in Colas (1995), "CCD3" for the one used by Mallama and described in Mallama (1992) and "IR-A" for the infrared array used at CRL, Tokyo and described in Souchay et al. (1992).

2. video mode uncooled CCD (unc.); images are recorded on a VCR as an analogic signal. Because of the large speed of acquisition (25 images per second, integrating time 0.02 second), the sensitivity is smaller than with a cooled CCD driven by a computer. So, a light intensifier may be used in front of the CCD target. The reduction is made by digitizing the images and by analyzing the numerical arrays of pixels. Such a digitizing technique is described by Arlot et al. (1989). These receptors are denoted "CCDVn" in the tables. Note than an intensified tube camera (SIT Vidicon) denoted "N" was also used in video mode.

3. the photographic technique may also be used as a twodimension receptor but the sensitivity is very low and the time constant very large. When used, such a technique has been denoted "PH" in the tables.

As an example, Fig. 2 shows how the reduction is performed for an event recorded using a two-dimensional receptor. This event has been observed at Meudon Observatory in very difficult local conditions with a video mode CCD camera. It was twilight and some light clouds were passing in front of Jupiter during the event. The raw signal shows evidently the decreasing twilight. After substracting the sky background, all the recorded objects show the variation of the transparency of the sky due to the clouds. Using a reference constant object, the light curve resulting from the reduction has the shape that we were looking for.

\subsection{Visual observations}

We give also visual lightcurves (denoted "V" in the data tables). They were obtained in most case using the Argelander method (Dumont \& Figer 1973). The magnitude scale is arbitrary and only the date of the minimum of the light curve is available from these data. Most of time, the time accuracy is better than one second of time. Very little information will be provided by the means of these light curves. However, in some cases, they may be helpful for analysis and comparison with the other ones. 
Table 1. Main sites of observation

\begin{tabular}{|c|c|c|c|c|c|c|c|c|c|}
\hline \multirow[b]{2}{*}{ Main observatories } & \multicolumn{4}{|c|}{ Longitude } & \multirow[b]{2}{*}{$\circ$} & \multicolumn{3}{|c|}{ Latitud e } & \multirow{2}{*}{$\begin{array}{l}\text { elevation } \\
\text { meters }\end{array}$} \\
\hline & $\mathrm{h}$ & $\mathrm{m}$ & $\mathrm{s}$ & & & & & & \\
\hline Barcelona (GEA-Spain) & 0 & 8 & 39.7 & $\mathrm{E}$ & 41 & 23 & 54 & $\mathrm{~N}$ & 19 \\
\hline Belogradchik (Bulgaria) & 1 & 30 & 42.0 & $\mathrm{E}$ & 43 & 37 & 35 & $\mathrm{~N}$ & 630 \\
\hline Beograd (Yugoslavia) & 1 & 22 & 3.0 & $\mathrm{E}$ & 44 & 48 & 12 & $\mathrm{~N}$ & 260 \\
\hline Berlin (Germany) & 0 & 53 & 40.0 & $\mathrm{E}$ & 52 & 32 & 0 & $\mathrm{~N}$ & 82 \\
\hline Bordeaux (France) & 0 & 2 & 6.6 & $\mathrm{~W}$ & 44 & 50 & 7 & $\mathrm{~N}$ & 73 \\
\hline Bowie, Maryland (U.S.A.) & 5 & 7 & 31.3 & $\mathrm{~W}$ & 38 & 58 & 55 & $\mathrm{~N}$ & 200 \\
\hline Brasopolis (Brazil) & 3 & 2 & 16.0 & $\mathrm{~W}$ & 22 & 31 & 6 & $\mathrm{~S}$ & 1870 \\
\hline Bucarest (Romania) & 1 & 44 & 23.1 & $\mathrm{E}$ & 44 & 24 & 50 & $\mathrm{~N}$ & 80 \\
\hline Calern (CERGA-France) & 0 & 27 & 41.2 & $\mathrm{E}$ & 43 & 45 & 17 & $\mathrm{~N}$ & 1282 \\
\hline Catania (Italy) & 0 & 59 & 55.0 & $\mathrm{E}$ & 37 & 41 & 30 & $\mathrm{~N}$ & 1725 \\
\hline Cluj-Napoca (Romania) & 1 & 34 & 23.0 & $\mathrm{E}$ & 46 & 42 & 48 & $\mathrm{~N}$ & 750 \\
\hline Devon Obs. (Canada) & 7 & 35 & 2.0 & $\mathrm{~W}$ & 53 & 23 & 26 & $\mathrm{~N}$ & 500 \\
\hline Jungfrau (Swizerland) & 0 & 31 & 56.4 & $\mathrm{E}$ & 46 & 32 & 53 & $\mathrm{~N}$ & 3578 \\
\hline Kakuda (Japan) & 9 & 24 & 0.0 & $\mathrm{E}$ & 38 & 0 & 0 & $\mathrm{~N}$ & 17 \\
\hline Kavalur VBO (India) & 5 & 15 & 19.6 & $\mathrm{E}$ & 12 & 34 & 32 & $\mathrm{~N}$ & 725 \\
\hline La Silla (ESO-Chile) & 4 & 42 & 55.1 & $\mathrm{~W}$ & 29 & 15 & 25 & $\mathrm{~S}$ & 2347 \\
\hline Mauna Kea (Hawaii, U.S.A.) & 10 & 21 & 7.2 & $\mathrm{~W}$ & 19 & 50 & 0 & $\mathrm{~N}$ & 4215 \\
\hline Meudon (France & 0 & 8 & 55.5 & $\mathrm{E}$ & 48 & 48 & 18 & $\mathrm{~N}$ & 162 \\
\hline Mollet (GEA-Spain) & 0 & 8 & 50.0 & $\mathrm{E}$ & 41 & 32 & 22 & $\mathrm{~N}$ & 70 \\
\hline Nice (France) & 0 & 29 & 19.1 & $\mathrm{E}$ & 43 & 43 & 17 & $\mathrm{~N}$ & 376 \\
\hline OHP (France) & 0 & 22 & 52.0 & $\mathrm{E}$ & 43 & 55 & 46 & $\mathrm{~N}$ & 665 \\
\hline Paris (France) & 0 & 9 & 20.9 & $\mathrm{E}$ & 48 & 50 & 11 & $\mathrm{~N}$ & 67 \\
\hline Pic-du-Midi (France) & 0 & 0 & 34.2 & $\mathrm{E}$ & 42 & 56 & 12 & $\mathrm{~N}$ & 2861 \\
\hline Reggio Calabria (Italy) & 1 & 2 & 36.4 & $\mathrm{E}$ & 38 & 6 & 25 & $\mathrm{~N}$ & - \\
\hline Reux (Belgium) & 0 & 20 & 21.8 & $\mathrm{E}$ & 50 & 14 & 43 & $\mathrm{~N}$ & 317 \\
\hline Rio de Janeiro (Brazil) & 2 & 52 & 53.6 & $\mathrm{~W}$ & 22 & 53 & 44 & $\mathrm{~S}$ & 33 \\
\hline Siding Spring (Australia) & 9 & 56 & 16.0 & $\mathrm{E}$ & 31 & 17 & 0 & $\mathrm{~S}$ & 1145 \\
\hline Tenerife (Canarian Islands,Spain) & 1 & 6 & 20.0 & $\mathrm{~W}$ & 28 & 15 & 0 & $\mathrm{~N}$ & 2400 \\
\hline Teramo (Italy) & 0 & 54 & 56.0 & $\mathrm{E}$ & 42 & 39 & 30 & $\mathrm{~N}$ & 388 \\
\hline Timisoara (Romania) & 1 & 24 & 55.0 & $\mathrm{E}$ & 45 & 44 & 15 & $\mathrm{~N}$ & 88 \\
\hline Tokyo (Japan) & 9 & 18 & 0.0 & $\mathrm{E}$ & 35 & 40 & 0 & $\mathrm{~N}$ & 58 \\
\hline Zoetermeer (The Netherlands) & 0 & 17 & 55.1 & $\mathrm{E}$ & 52 & 04 & 11.6 & $\mathrm{~N}$ & 5 \\
\hline
\end{tabular}

Note that most of the observers belong to the GEOS association (Groupe Etudes et Observations Stellaires).

\section{Lightcurves reduction procedure}

Only relative photometry was made since the air mass was very large most of time. The sky-background was carefully recorded several times, especially during daylight in order to be eliminated. When possible, the flux of a reference object was recorded (a star or, most of time, another satellite which is acceptable because of the short duration of the events). This made possible the observations even when small clouds or fog were present. Figure 2 gives an example of a complete reduction using the sky-background and a reference object for an observation made at Meudon Observatory during twilight with some light clouds in front of Jupiter (cf. Sect. 3). This observation has been made using a CCD target in video mode. The final lightcurve demonstrates the power of a two-dimensional sensor which records the sky background and a reference satellite at the same time as the occulted or eclipsed satellite. The determination of the date of the minimum of light and of the value of the magnitude drop was obtained from a fit of the lightcurve with a sample polynomial. The errors on these determinations are also given.

\section{The catalogue - Discussion of the data}

\subsection{The data}

At the end of this paper, the data are given. For each observed event, Tables 3 give the determined date of the minimum of light and the magnitude drop. In these tables, we also give the corresponding calculated data using 3 ephemerides and 2 algorithms. Both ephemerides are issued from Lieske's work (1977): G-5 ephemerides are fitted on photographic observations made from 1891 to 1978 (Arlot 1982), E-3 ephemerides are fitted on 


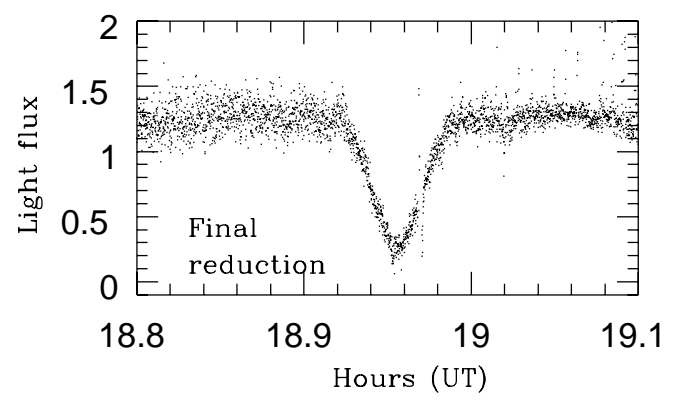

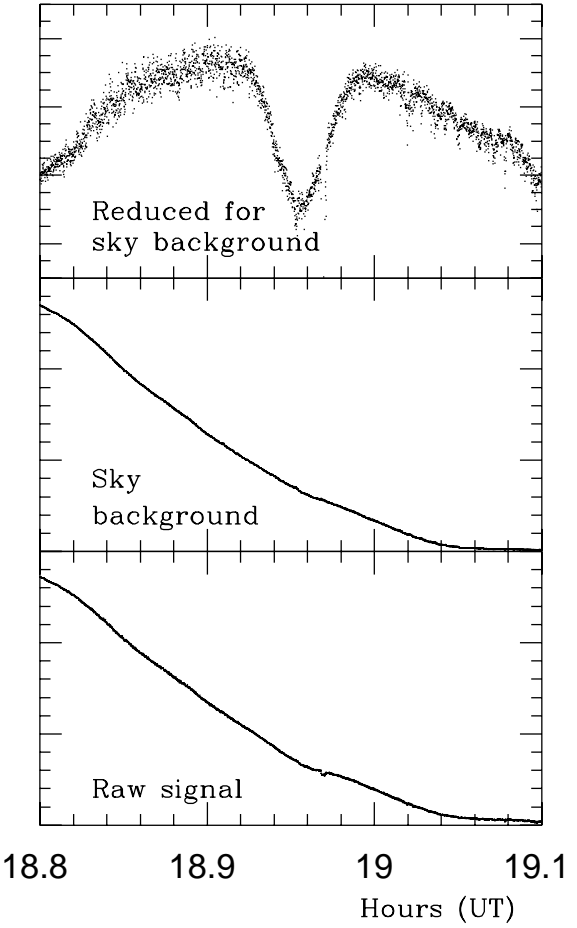

Eclipsed satellite

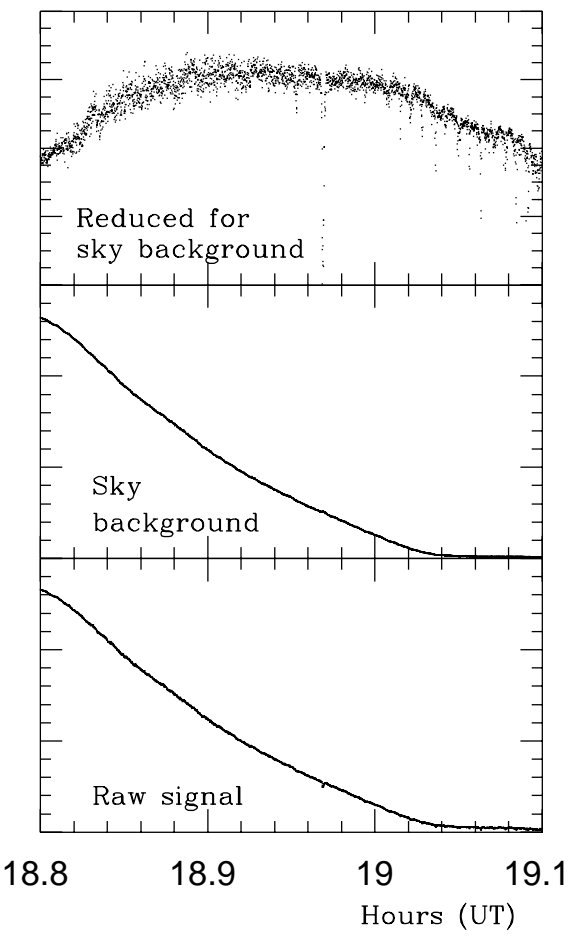

Reference satellite

Fig. 2. Observation of the eclipse of J2 by J1 at Meudon Observatory on April 22, 1991

observations of several kind mostly eclipses by Jupiter made from 1652 to 1983 (Lieske 1987) and E-2 ephemerides are fitted on recent photographic data and old eclipses (Lieske 1980). Two algorithms were used (denoted (1) and (2) in the catalogue). The first one supposes that both involved satellites are uniform disks and the second one takes into account the phase defect and uses the Hapke's law (Hapke 1986) of diffusion of light to describe the apparent disks of the satellites whose surfaces are always supposed to have an uniform albedo (Thuillot \& Morando 1990; Descamps 1992). For each event and each site of observation, we give also:

1. the $(\mathrm{C}-\mathrm{O})$ of the observation;

2. the type of telescope used in column "Inst." (denoted "T" for a reflector and "L" for a refractor);

3. the aperture of the telescope in centimeters;

4. the type of receptor in column "Recept." (cf. Sect. 3);
5. the elevation of Jupiter and the Sun upon the horizon in the two next columms;

6. the apparent distance from the involved satellites to the center of Jupiter in jovian radii;

7. the observational conditions in column "Obs. cond.": [1] means very good conditions; [2] means acceptable and $[3]$ very difficult conditions;

8. the eventual filter used during the observations in column "Filter";

9. the time-sampling of the light curve in seconds of time.

10. the size of the diaphragm when used;

11. the satellites in the diaphragm, i.e. the satellites, the global magnitude drop of which was observed.

Figure 3 gives all the lightcurves in the same chronological order as the Tables. The light curves reported from VBO (Kavalur) correspond to the light variation of the occulted or eclipsed satellite. The contribution of the occulting or eclipsing satellite were determined before and 
after the observation and were substracted from the total flux so that, for this site, the observed lightflux drops are not comparable with the others.

These data and light-curves are available for anyone who is interested through the electronic network on the WEB server (http://www.bdl.fr) and on the ftp anonymous server (ftp://ftp.bdl.fr, directory: /pub/NSDC/jupiter/pheno_mut/1991) of Bureau des longitudes.

Table 2. Receptors used for the observations

\begin{tabular}{|c|c|}
\hline $\begin{array}{l}\text { Code as } \\
\text { given in } \\
\text { the tables }\end{array}$ & Description \\
\hline & Single channel receptors \\
\hline $\begin{array}{l}\text { PM1 } \\
\text { PM2 } \\
\text { PM3 } \\
\text { PM4 } \\
\text { PM5 } \\
\text { PM6 } \\
\text { PM7B } \\
\text { PM7R } \\
\text { PM8 } \\
\text { PM9 } \\
\text { PM10 } \\
\text { PM11 } \\
\text { PM12 } \\
\text { PM13 } \\
\text { PM14 } \\
\text { PM15 } \\
\text { PM16 } \\
\text { PM17 } \\
\text { PMB } \\
\text { PMV } \\
\text { PMR } \\
\text { PMK } \\
\text { S20 } \\
\text { S20R } \\
\text { S11 } \\
\text { PMIR } \\
\text { PM }\end{array}$ & $\begin{array}{l}\text { photom. EMI9502B (Bucarest, Beograd) } \\
\text { photomultiplier EMI9789QA (Belogradchik, Catania) } \\
\text { photom. Quantacon RCA 31036 Ga-As (ESO) } \\
\text { photom. Hamamatsu EMI6256SA S-11 (ESO) } \\
\text { PIN photodiode OPTEC SSP13 (Essen, Holtsville, GEA) } \\
\text { photom Hamamatsu R647 1P21 (Kakuda) } \\
\text { photom. RTC 2020 (Nice) } \\
\text { photom. Hamamatsu } 6375 \text { (Nice) } \\
\text { photom. EMI9558QB (Cluj-Napoca) } \\
\text { photom. RCA 4840 (Paris) } \\
\text { photom. EMI9789QB (Reggio Calabria) } \\
\text { photom. RCA 6199 (Rio de Janeiro) } \\
\text { photom. attached to Siding Spring tel. } \\
\text { photom. EMI9862QB (Timisoara) } \\
\text { DOAA photod. SSP (Zoetermeer) } \\
\text { photom. Hamamatsu 943-02 (Brasopolis) } \\
\text { photom. RCA C31034A (Mauna Kea) } \\
\text { photom. HPO(1P21) or OPTEC SSP5 } \\
\text { photom. TELOC II channel B (Calern) } \\
\text { photom. TELOC II channel V (Calern) } \\
\text { photom. TELOC II channel R (Calern) } \\
\text { photom. EMI9658R (Kavalur) } \\
\text { photom. EMI9658B S20 cathode (Kavalur) } \\
\text { photom. EMI9658R S20R cathode (Kavalur) } \\
\text { photom. with S11 cathode (Jungfrau) } \\
\text { photometer IRPHOT2 1.5 micrometers (OHP) } \\
\text { unidentified photomultiplier }\end{array}$ \\
\hline PM & Two-dimensional receptors \\
\hline $\begin{array}{l}\text { CCD1 } \\
\text { CCD2 } \\
\text { CCD3 } \\
\text { IR-A } \\
\text { CCDV1 } \\
\text { CCDV2 } \\
\text { CCDV3 } \\
\text { CCDV4 } \\
\text { CCDV5 } \\
\text { CCDV6 } \\
\text { CCDVX } \\
\text { N } \\
\end{array}$ & $\begin{array}{l}\text { c. CCD camera with TH7852 target (Bordeaux) } \\
\text { c. CCD camera Astriane (Pic du Midi) } \\
\text { c. CCD camera with TC-211 chip (Bowie) } \\
\text { cooled Rockwell HgCdTe } 128 \times 128 \text { array } 2.2 \mu \mathrm{m} \text { (CRL) } \\
\text { video mode unc. CCD SBIG } \\
\text { video mode unc. CCD Sony ICX021 } \\
\text { video mode unc. CCD Imaintel } \\
\text { video mode unc. CCD with intensifier } \\
\text { video mode unc. CCD Panasonic } 0.5 \text { lux } \\
\text { video mode unc. CCD Philips } 56470 \text { NXA 1011/01 } \\
\text { video mode unc. CCD MXRII HCS Vision Techn. } \\
\text { video mode unc. SIT Vidicon (Nocticon) }\end{array}$ \\
\hline & Other types of receptors \\
\hline & $\begin{array}{l}\text { visual observation using Argelander method } \\
\text { photographic observation }\end{array}$ \\
\hline
\end{tabular}

\subsection{Discussion}

In this paper, we do not intend to make a complete analysis of the data. These data may be analyzed for astrometric purpose as well as for planetologic interpretation. However, it is interesting to compare the different predictions and also the difference between the midevent defined as the closest approach of the two satellites -case (1)and as the minimum of light -case (2)-. Note that some of the data presented in this catalogue for comparison with the other results have been analyzed yet by Mallama (1992), Froeschlé et al. (1992), Le Campion et al. (1992), Descamps et al. (1992) and Souchay et al. (1992).

Because of the very different time constants used for each observation, the quality of each lightcurve may be judged either with the value of the errors on the determinated parameters (time of the minimum of light and magnitude drop) or with the appearance of the lightcurve itself. The error bars are calculated as follows: - the error on the magnitude drop comes from the standard deviation from the fit to the model light curve (this explains that the error will decrease when the number of points decreases by averaging several successive points); - the error on the date of the minimum is deduced from the error on the magnitude drop combined with the speed of the decrease of the magnitude during the event (this explains that this error depends on the number of points, on the integrating time and on the depth of the light curve). Because of that, the errors bars may be compared only between events made with the same time constants and, preferably, with the same equipment. One will notice some bad determinations of the magnitude drops: this comes from the difficult conditions in which the corresponding observations have been made (small elevation above the horizon, twilight, vicinity of Jupiter or bad meteorological conditions). So, the two informations, time of the minimum of light and value of the magnitude drop do not have to be mixed in a single positional $(\mathrm{O}-\mathrm{C})$. In a first step, the observed time of the minimum of light is more confident for theoretical studies.

We note that a good model is needed to fit the observed light curves, in order to determine accurate times of minimum light and magnitude drop. Such a model can also allow to observe beginning and ending times of the event to be determined. The predictions based on such a model can be directly compared to observations. Finally, a reduction that accurately models the albedo features and limb darkening of the satellites will give the best relative positions of the two satellites at the time of the event (Descamps et al. 1992; Mallama 1991; Mallama 1992).

\section{Conclusion}

The data reported in this paper show how useful a campaign to observe mutual events can be. Unfortunately the data from a few observers could not be used. In order to 
avoid such accidents in future campaigns, observers are reminded that:

1. the time in UTC is essential and we have to be sure of the scale of time within at least half-a-second;

2 . in order to use observations made in difficult conditions (small clouds, ...) the recording of a reference object and of the sky-background simultaneously with the eclipsed or occulted satellites is absolutely necessary. Many lightcurves are of good quality only because of such recordings (cf. Fig. 2) and some have been rejected because of the absence of such recordings.

After the PHEMU91 campaign, we look forward for future campaigns: first, we will make a special effort to observe the events of the satellites of Saturn in 1995-1996. They are fainter than the Galilean satellites and larger telescopes should be used; however these events occur only every 15 years which justifies the international campaign we organize and called PHESAT95.

Second we will apply our techniques to observe the mutual events which will occur during the next favorable period in 1997: we will use the experience of the 1991 observations in order to improve the data obtained. If you are interested in joining us for the next campaigns and if you like to receive technical notes in order to help you to observe, please, write to us.

Acknowledgements. These observations have been made possible thanks to the CNRS (Centre National de la Recherche Scientifique), the INSU (Institut National des Sciences de l'Univers) and the CNES (Centre National d'Etudes Spatiales) through the PNP (Programme National de Planétologie) who supports the PHEMU91 campaign, the Bureau des Longitudes and the Observatories where observations were made. We thank amateur astronomers J. Juan-Samso, S. Torrell, F. Gallego, J. Blasco, R. Guarro, C. Gallart, J. Foch, J.V. Piqueras, F. Campos and D. Fernandez for observing these events. We also wish to thank the staff of the observatories where observations were made, especially M.M. Bretagne, Gravallon, Mevolhon, Leydet, Rambaud, Rau and Richaud from Observatoire de Haute-Provence for their help during this campaign and Mrs. Derouazi and Baron for the type-writing of the tables.

\section{References}

Arlot J.-E., 1982, A\&A 107, 305

Arlot J.-E., 1990, A\&A 237, 259

Arlot J.-E., Bernard A., Bouchet P., et al., 1982, A\&A 111, 151

Arlot J.-E., Thuillot W., Barroso Jr. J., et al., 1992, A\&AS 92, 151

Arlot J.-E., Thuillot W., Colas F., Allet Ch., Vu D.T., 1989, Ce lest. Mech. 45, 129

Blanco C., Di Martino M., Ferrari W., 1991, AJ 101, 2262

Briot D., 1987, Supp. Ann. Phys. 12, 27

Burchi R., Di Paolantonio P., 1987, Supp. Ann. Phys. 12, 67

Colas F., 1995, Proceedings of the PHESAT95 Workshop, Supp. Ann. Phys. (in press)

Descamps P., 1992, thèse de l'Observatoire de Paris

Descamps P., Arlot J.-E., Thuillot W., et al., 1992, Observations of the volcanoes of Io, Loki and Pele, made in 1991 at the ESO during an occultation by Europa, Icarus 100,235

Dumont M., Figer A., 1973, L'Astronomie 87, 141

Froeschlé M., Helmer G., Meyer C., 1988, A\&A 189, 277

Froeschlé M., Meyer C., Mignard F., 1992, A\&A 262, 308

Goldstein S.J. Jr., Jacobs K.C., 1986, AJ 92, 199

Gomez-Forrellad J.M., 1987, Supp. Ann. Phys. 12, 79

Gouiffes Ch., Bouchet P., Schmider F.X., 1987, Supp. Ann. Phys. 12, 97

Hapke B., 1986, Icarus 67, 264

Le Campion J.F., Montignac G., Chauvet F., Colin J., Desbats J.M., Dourneau G., Rapaport M., 1992, A\&A 266, 568

Lieske J.H., 1977, A\&A 56, 333

Lieske J.H., 1980, A\&A 82, 340

Lieske J.H., 1987, A\&A 176, 146

Mallama A., 1991, Icarus 92, 324

Mallama A., 1992, Icarus 97, 298

Mallama A., Caprette D.S., Nelson P., Park J., Collins D.F., Vojtisek-Lom M., 1994, Icarus 107, 212

Souchay J., Hiromoto N., Takami H., Descamps P., Aruga T., 1992, A\&A 264, 314

Thuillot W., Morando B., 1990, Note Scientifique et Technique du Bureau des Longitudes S030, Paris 